\title{
Development of Composite Propeller Blade Model for High Velocity Impacts on Aircraft Fuselage using Finite Element Analysis
}

\author{
Vasilis Votsios $^{1 *}$, Esteban Martino-Gonzalez ${ }^{1}$ and Jorge Lopez-Puente ${ }^{2}$ \\ ${ }^{1}$ Airbus Operations S.L., Getafe, Spain \\ ${ }^{2}$ University Carlos III of Madrid, Leganés, Madrid, Spain
}

\begin{abstract}
An open rotor blade failure and release event can result in a high energy impact on an aircraft fuselage that can reduce the strength of the structure and challenge the safe continuation of the flight and landing. This work highlights the development of a numerical approach and methodology in order to improve the assessment of the damage predictions of a composite propeller blade impact against the fuselage of an aircraft to be able to estimate a minimum thickness of shielding for the full protection of the airframe. A number of dynamic simulations were carried out, from rigid up to deformable and frangible projectiles at different angles of incidence, varying the material and the thicknesses using Abaqus/Explicit. The finite element (FE) models for blade and target were calibrated and validated separately allowing to capture the right behavior and failure modes. Impact tests of partial blade fragments against stiffened composite panels were correlated with simulations and the obtained results show a good agreement regarding deformations and delaminated area. Finally, a full blade FE model was generated and used for the fuselage impact numerical analysis. This was done within the frame of the Open Rotor project funded by Clean Sky European research programme.
\end{abstract}

\section{Introduction}

Key for the development of the next generation aircraft is the implementation of advanced green technologies, focusing principally on the efficiency improvement and also on the reduction of emissions. Within the European Clean Sky framework, Airbus had the opportunity to mature innovative ideas and study the viability of an aircraft concept with open rotor engine mounted on the rear end (Fig. 1). An open rotor engine is defined as a turbine engine featuring contra-rotating fan stages not enclosed within a casing [1]. However, one of the main certification risks for a Contra-Rotating Open Rotor (CROR) is the safety level demonstration and the preclusion of any hazard or catastrophic effects during an Open Rotor Blade Release (ORBR) event. This could potentially result in a blade impact on the airframe reducing the strength of the structure, to cross engine debris exposure or to collateral damage on adjacent blades and therefore challenge the safe continuation of flight and landing.

* Corresponding author: vasilis.votsios@airbus.com 


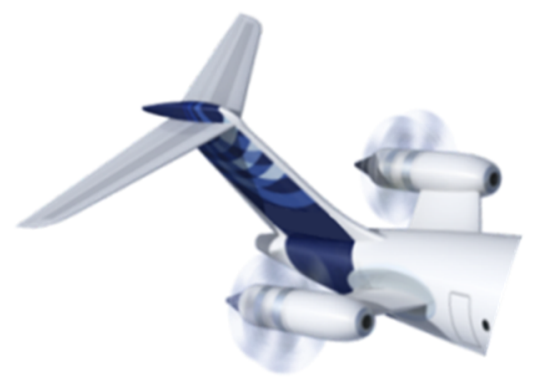

Fig. 1. Illustration of CROR concept

This work describes the development of a representative full size composite aerofoil nonlinear finite element (FE) model that was used for the analysis of the ORBR effects and impact behaviour and hence to develop shielding solutions and assess their level of effectiveness regarding the airframe protection. Several studies were carried out including physical tests and numerical simulations with the aim to develop predictive finite element models with failure criteria and validate the maturity of the applied methodology following the building block approach (Fig. 2).

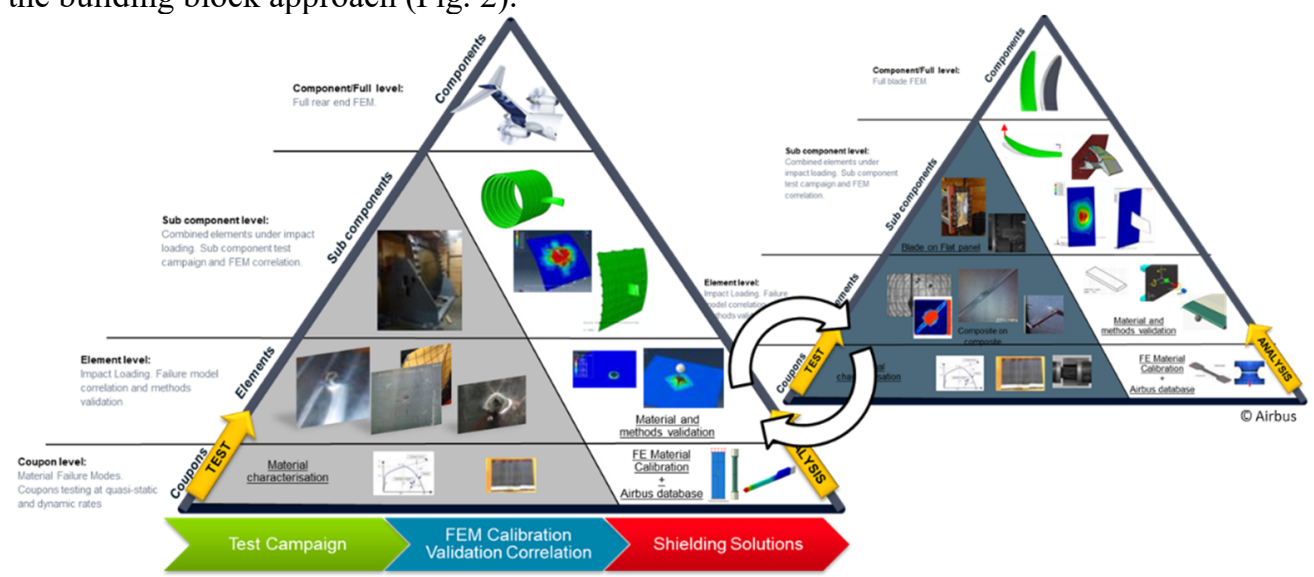

Fig. 2. Building block approach: Test pyramid to validate FE models

In parallel with the CROR innovative shielding solutions development, the projectile definition plays an important role for the overall damage assessment and for the better understanding of the failure modes that take place in such a high energy impact event. As it can be seen in Fig. 2 a separate test pyramid was followed for the blade modelling, which was subsequently divided into two phases. Firstly, segments from a generic turboprop propeller blade were employed for impact tests on flat and curved panels and the test results were used for the calibration, correlation and validation of the modelling methodology. In continuation a full aerofoil model was generated for numerical simulations of higher level of complexity. In a second phase, the turboprop full blade FE model was replaced by a generic CROR blade. The influence of the different blade architectures under the same impact conditions was analysed. In addition, the model was qualitatively correlated with the blade release full scale impact test of NASA Glenn Research Center and the Naval Air Warfare Center (NAWC) China Lake [2] related to the work of The Federal Aviation Administration (FAA) and the European Aviation Safety Agency in order to determine the certification requirements for open rotor engines. 


\section{Model development and validation}

The model preparation was done in Abaqus/CAE and Altair Hypermesh and all numerical simulations were performed with Abaqus/Explicit V6.13-2. A description of the projectile and target modelling is given in following sections.

\subsection{Projectiles modelling}

\subsubsection{Blade fragments}

Two type of fragments were used for the impact tests. These correspond to a tip section and a centre section from a turboprop blade (Fig. 3).
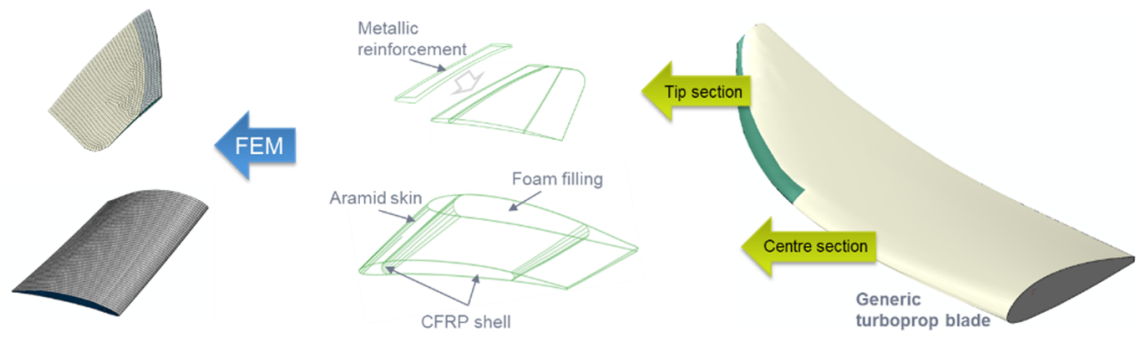

Fig. 3. Generic turboprop blade and fragments section

A typical architecture of a composite blade consists of an aramid fibre (woven fabric) outer skin, carbon fibre-reinforced polymer composite (CFRP) spars, foam core inserts and a metallic reinforcement on the leading edge. The aramid skin, CFRP spars and the metallic reinforcement were meshed with four-node, reduced integration shell elements (S4R), while the foam filling material was meshed with eight-node, reduced integration solid elements (C3D8R). The interface between the parts (i.e. skin and foam, spars and foam, etc.) were modelled by using coincide nodes or tied interfaces.

\subsubsection{Full blade}

In the full blade the aramid skin was meshed with continuum shell elements (SC8R). This was done because of numerical errors and excessive distortion issues of the conventional shell elements (S4R) during the simulation. Also, the foam modelling was changed from solid to tetrahedral elements in order to achieve a smooth thickness transition along the span and chord of the blade. The CFRP spars and the metallic reinforcement were meshed with S4R elements. The interface between parts (i.e. skin and foam, spars and foam, etc.) were modelled by using coincide nodes. The FE models are shown in Fig. 4.
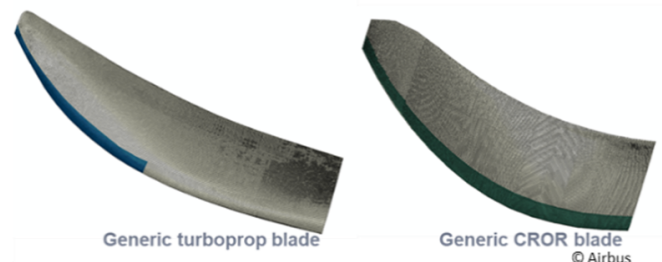

Fig. 4. Generic turboprop and CROR full blade FE models 


\subsection{Targets modelling}

\subsubsection{Flat panels}

Impact tests of aluminium and uni-directional (UD) CFRP flat rectangular panels of $350 \mathrm{~mm}$ $\mathrm{x} 700 \mathrm{~mm}$ of different thicknesses were used for the model validation. The aluminium panels were modelled using reduced integration conventional shell elements (S4R) and the composite ones with tri-dimensional, reduced integration continuum shell elements (SC8R).

\subsubsection{Curved stiffened panel}

The next impact test level consisted of a representative fuselage section of a $2 \mathrm{~m}$ x $2 \mathrm{~m}$ CFRP curved panel with stringers and frames (Fig. 5). The composite panel was meshed with continuum shell elements (SC8R), while the frames and stringers were modelled with conventional shell elements (S4R). A layer of cohesive elements was applied at the centre of the panel as a simplified approach for capturing delamination effects. Cohesive elements were also used for the bonding of the stringers to the panel. The frames were connected to the curved panel by means of fasteners modelled as connector elements (CONN3D2).

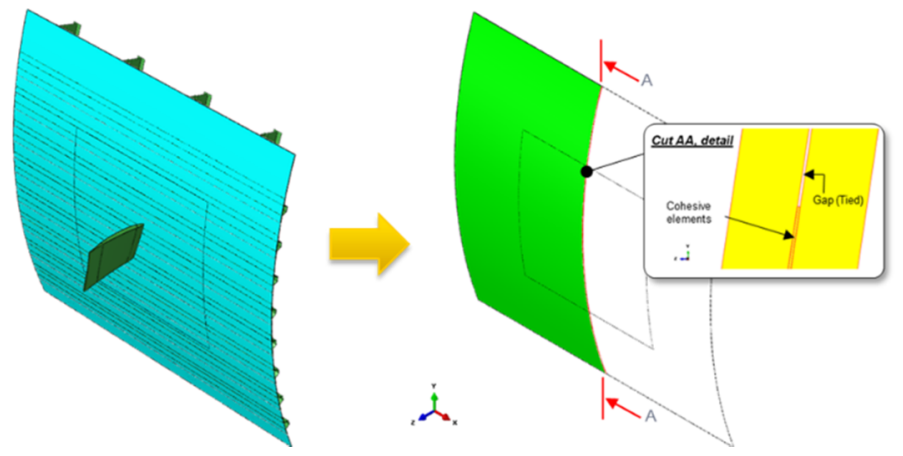

Fig. 5. Curved stiffened CFRP panel

\subsection{Material definition}

UD composite lamina-type materials (i.e. flat and curved panels) were modelled using the Hashin damage initiation model coupled with damage evolution defined in a fracture energycontrolled manner, with different values of fracture energy for different fracture modes (longitudinal or transverse tension or compression). For the woven fabrics the fabric material model available as a built-in VUMAT user subroutine in Abaqus/Explicit (ABQ_PLY_FABRIC) was used. The foam was idealised with elastic-perfectly plastic mechanical behaviour. A ductile damage model was used to simulate possible fracture with damage initiation (strain-controlled) and damage evolution (displacement-controlled). The metallic materials were either described by Johnson-Cook (flat panel) or tabular (frames) hardening law with damage initiation and damage evolution. Fasteners were modelled with elastic, plastic and damage behaviour for the six degrees of freedom. The constitutive behaviour used for the cohesive elements was described by means of a traction-separation law with uncoupled elasticity before damage initiation, followed by linear softening. Damage initiation was defined in terms of cohesive strength by using a quadratic stress-based criterion and the damage evolution as fracture energy for pure traction and shear modes with an exponent for the power-law criterion. The material data used for the simulation models are based on Airbus material characterisation test campaigns. 


\section{Results}

The blade model was validated through the test pyramid levels using impact energies from $10 \mathrm{~kJ}$ to $110 \mathrm{~kJ}$, corresponding to the fragments and to different full aerofoil configurations.

\subsection{Flat panel tests}

In the element level of the test pyramid, a number of flat panels were tested and used for the correlation and validation of the target materials and blade fragments. Aluminium and UD CFRP specimens were impacted with a steel spherical projectile to define the ballistic curves (Fig. 6).
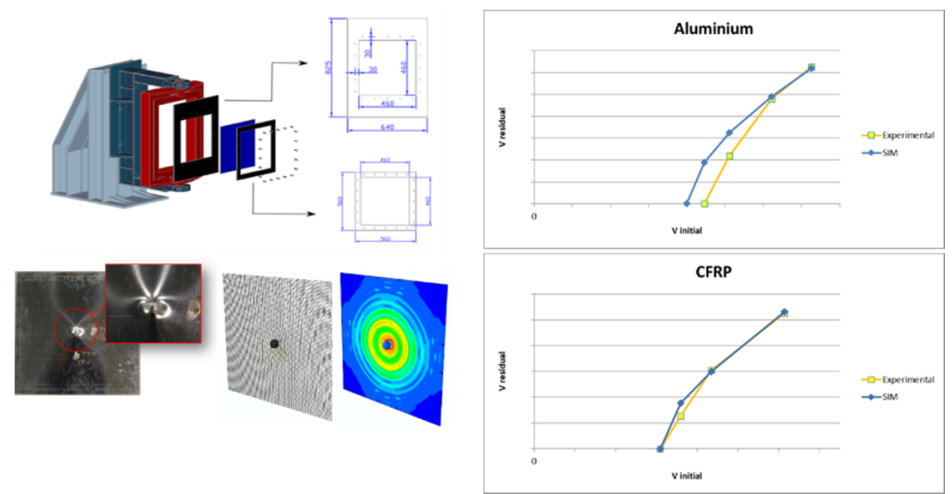

Fig. 6. Flat panel test setup and FEM correlation.

In general, a good correlation was obtained between test results and simulations, showing an appropriate modelling approach for ballistic and damage predictions. The second part within the element level of the test pyramid involved impacts of a blade centre section fragment against aluminium and UD CFRP panels of different thicknesses for the purpose of deformable up to rigid target impacts, allowing for the assessment of the fragmentation and general behaviour of the projectile (Fig. 7).
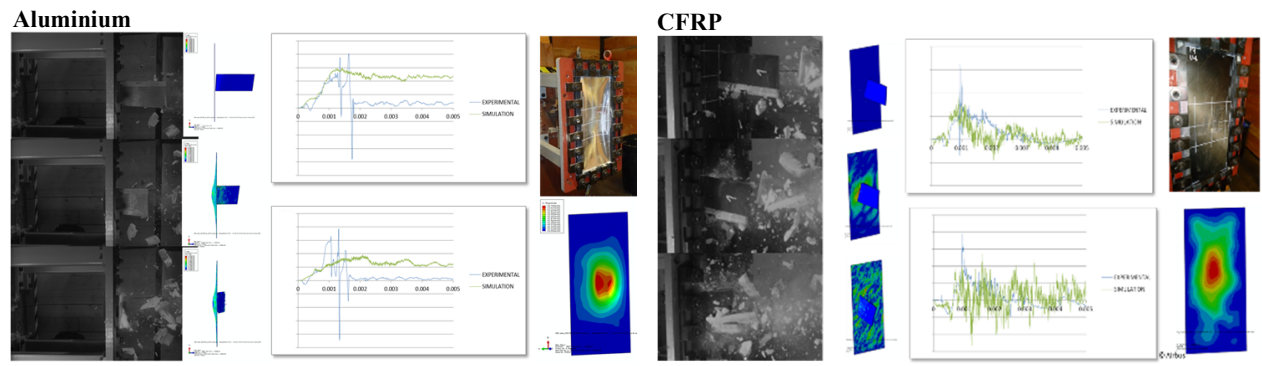

Fig. 7. Blade fragment impact on flat panel: test and FE analysis correlation

\subsection{Curved panels}

Impact tests of a blade centre and tip fragments against curved stiffened panels were carried out at different incident angles, at mid bay and near the frame locations. A summary of test results and FEM correlation is shown in Fig. 8 and Fig. 9. For the case of a normal impact with a centre fragment projectile at $123 \mathrm{~m} / \mathrm{s}$ there was no penetration. However, localised damage was observed on the panel near to the frames. The impact sequence was captured well by the FE simulation with some underestimation in the cracking prediction. 
On the other hand, although slightly overestimated, there was a good prediction of the delaminated area and stringer debonding. Also, a good correlation can be seen at the strain gauge correlation between test and simulation at locations near the impact point (Fig. 8). Similar results are shown in Fig. 9 for the case of impact near to the frame, which led to the penetration of the specimen.

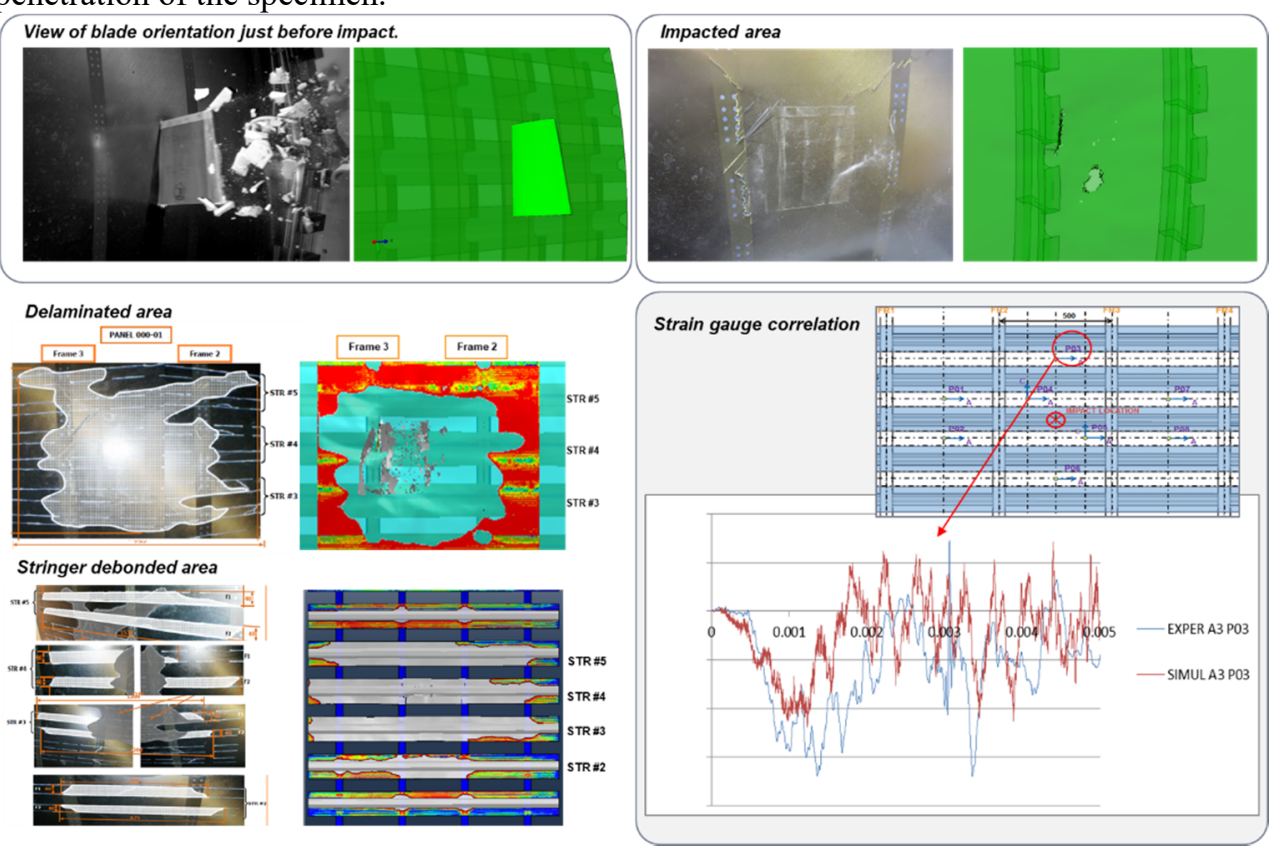

Fig. 8. Centred normal impact of blade fragment on curved panel: test and FE analysis correlation
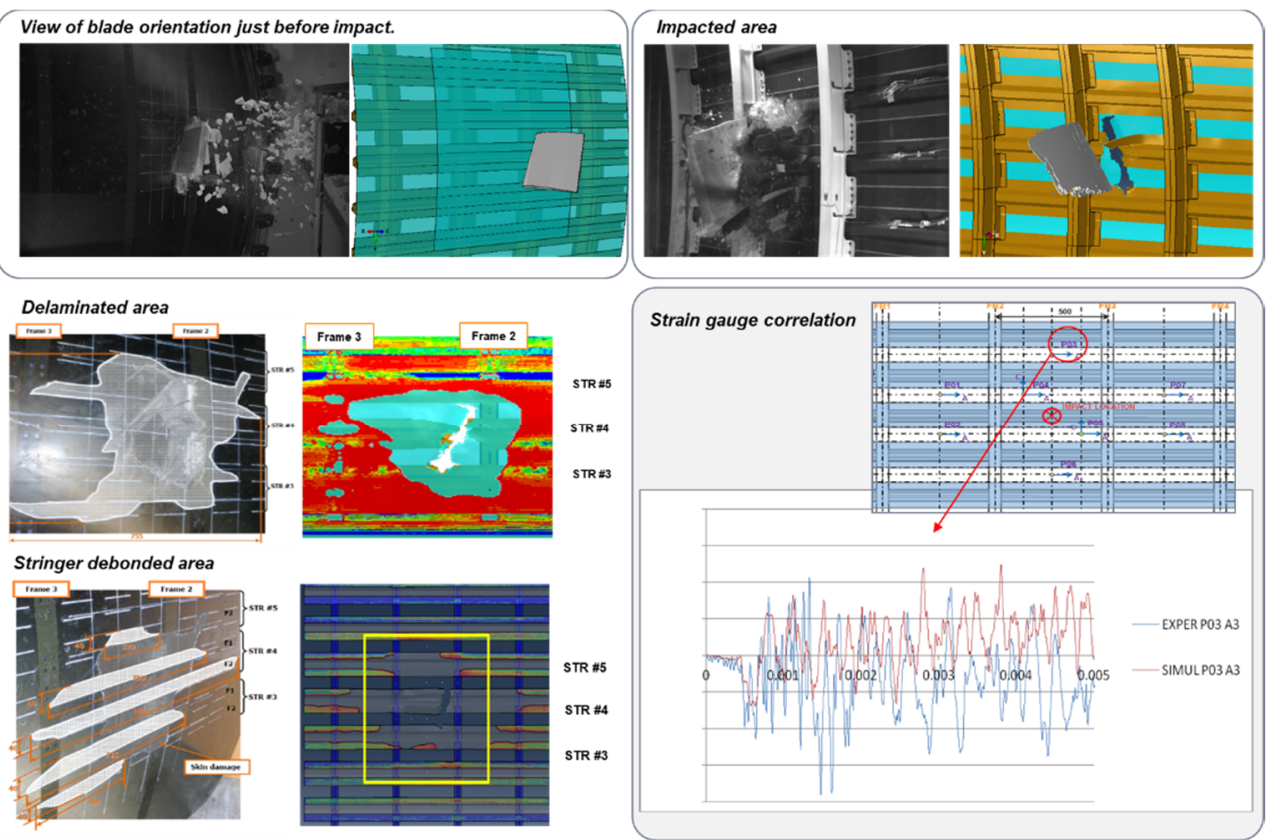

Fig. 9. Near to frame impact of blade fragment on curved panel: test and FE analysis correlation 
Blade tip-section, before and after impact is shown in Fig. 10.
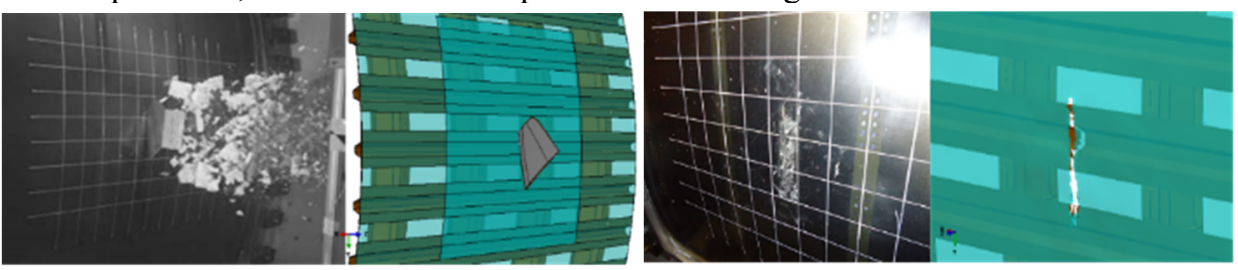

Fig. 10. Blade tip section impact test and simulation results

\subsection{Comparison with NASA open rotor full-scale test}

In order to verify the global behaviour of the full blade FE model, a qualitative correlation was carried out with the impact test conducted by the Naval Air Warfare Center Weapons Division (NAWCWD) Weapons Survivability Lab (WSL) [2], where $6.85 \mathrm{~kg}$ blades were released at $162.5 \mathrm{~m} / \mathrm{s}(91 \mathrm{~kJ})$ against triaxial braided composite panels of different thicknesses (Fig. 11). Simulation models were built using the CROR full blade model and a composite panel of UD CFRP (the same as in the blade fragments correlation).
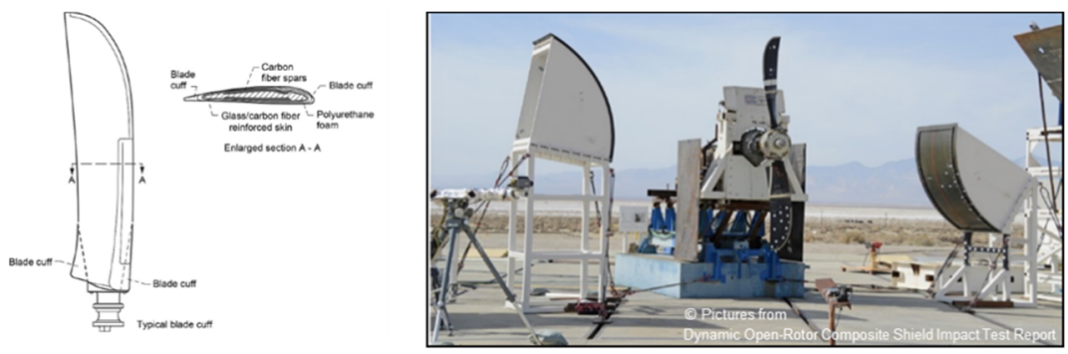

Fig. 11. Typical blade (left) and test rig setup (right), from NASA Glenn Research Center and

NAWC China Lake [2]

It must be noted that the blade FE model was of similar weight and architecture as the one in the NASA and NAWC test. Also, impact test results of metallic spherical projectile on triaxial braided panels of different thicknesses (test pyramid, element level) showed a similar ballistic tendency compared to the specific UD CFRP shielding used in this work. This allowed to derive an equivalency factor for the qualitative correlation between Airbus UD panel with CROR blade against the full-scale impact test. Finally, the numerical analysis showed a good agreement with the full-scale test. Similar blade fragment survived after the impact and the UD panel thickness, for no penetration, was slightly higher, verifying the same tendency as in the element-level tests results (Fig. 12).
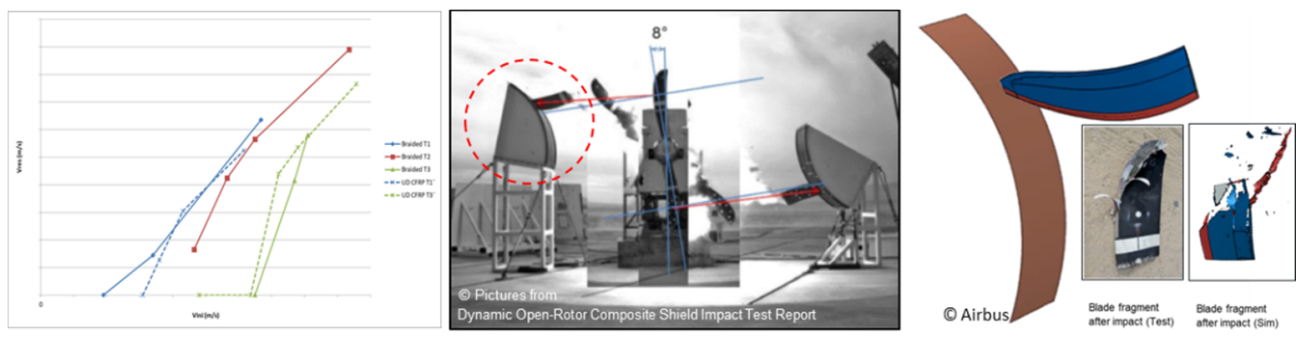

Fig. 12. Ballistic curves, impact sequence and numerical analysis (from left to the right) 


\section{Conclusions}

This work outlines the development of a full blade finite element model with the purpose to be used in the damage assessment of an airframe followed by a blade release event, to provide support to the studies for innovative shielding configurations and even optimise the blade design by means of simulation. The modelling methodology of the blade was based on a step by step validation following a building block approach, allowing for the model calibration and improvement of modelling techniques. Validation was performed for both projectile and targets in a parallel manner and despite the assumptions and limitations in the modelling, at the end a good correlation of the FE simulations with experimental data was achieved, providing confidence for the damage and penetration predictions. Under the scope of future aircraft developments, the validated models are enabling the evaluation of innovative shielding solutions (related to blade release) and the estimation of the associated weight penalty impact at aircraft level.

\section{Acknowledgement}

This was work was partially funded by the European Commission within the framework of the EU Research Programme Clean Sky. The funding is gratefully acknowledged.

\section{References}

1. EASA, NPA, Open rotor engine and installation, RMT.0384 (2015)

2. S. Seng, C. Frankenberger, C. R. Ruggeri, D. M. Revilock, J. M. Pereira, K. S. Carney, W. C. Emmerling, Dynamic Open-Rotor Composite Shield Impact Test Report, NASA/TM-2015-218811, DOT/FAA/TC-15/22 (2015) 